Supporting information for

\title{
Flexible high-efficiency CZTSSe solar cells on diverse flexible substrates via an adhesive-bonding transfer method
}

Jung-Hong Min, ${ }^{1,2, 广}$ Woo-Lim Jeong,,${ }^{1,2, \dagger}$ Kiyoung Kim, ${ }^{1}$ Je-Sung Lee, ${ }^{1}$ Kyung-Pil

Kim, ${ }^{1}$ Jihun Kim, ${ }^{1}$ Myeng Gil Gang, ${ }^{3}$ Chang Woo Hong, ${ }^{3}$ Jin Hyeok Kim, ${ }^{3}$ and DongSeon Lee ${ }^{1,2, *}$

${ }^{1}$ School of Electrical Engineering and Computer Science, Gwangju Institute of Science and Technology, Gwangju 61005, South Korea

${ }^{2}$ Research Institute for Solar and Sustainable Energies, Gwangju Institute of Science and Technology, Gwangju 61005, South Korea

${ }^{3}$ Optoelectronic Convergence Research Center, Department of Materials Science and Engineering Chonnam National University, Gwangju 61186, South Korea

$\dagger$ These authors contributed equally to this work.

* Corresponding author. Tel.: +82 62 7152248; fax: +82 627152204

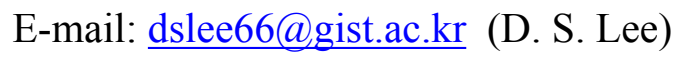




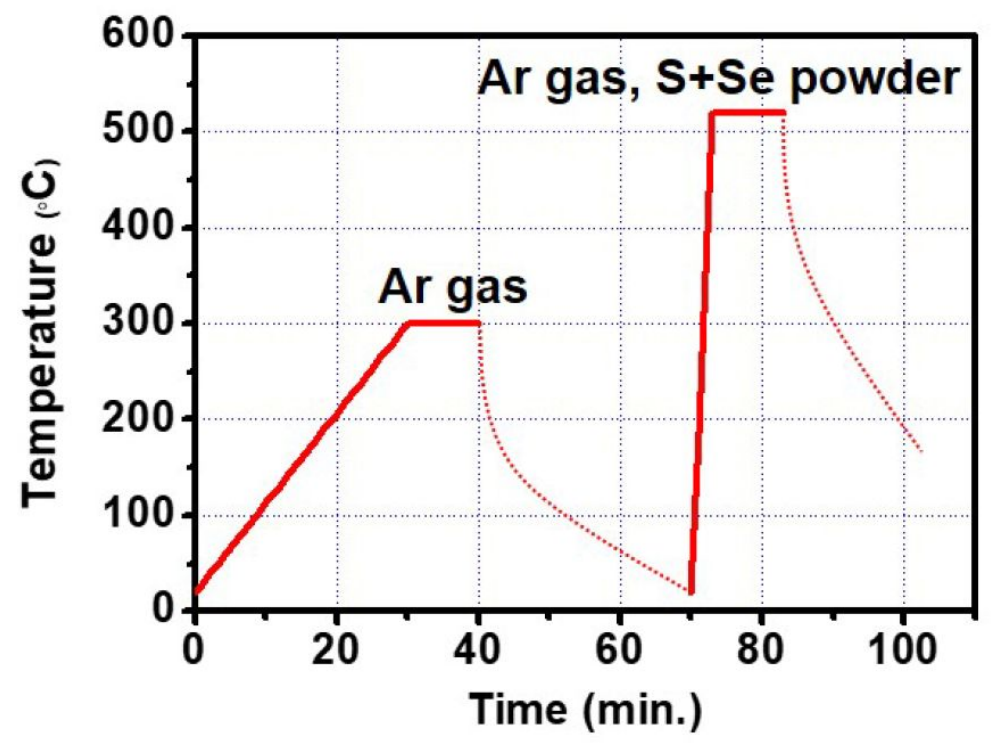

Figure S1. Profiles of temperature and ambient conditions for thermal annealing [1].

(a)

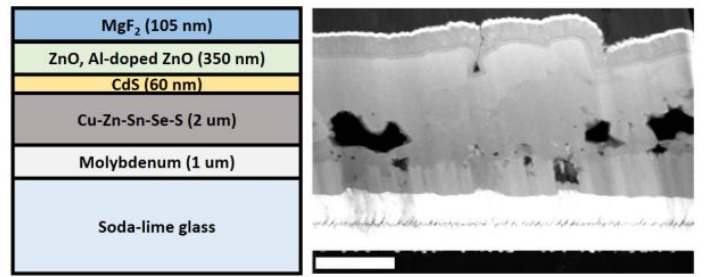

(b)

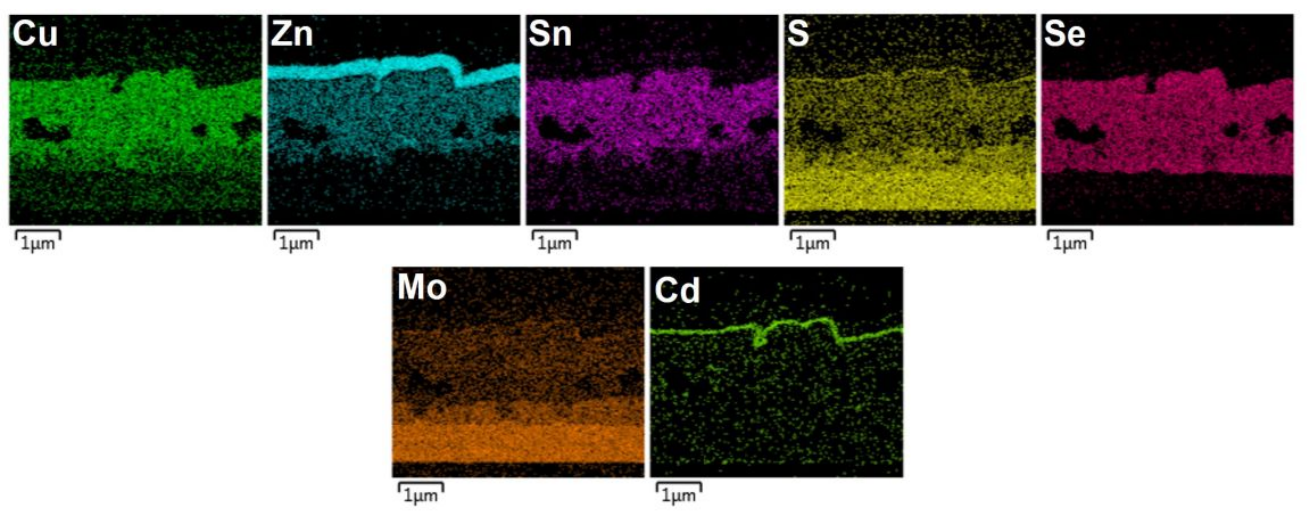

Figure S2. (a) Schematic of CZTSSe solar cell and its TEM image. The scale bar is 1 $\mu \mathrm{m}$. (b) EDX mapping for TEM related to $\mathrm{Cu}, \mathrm{Zn}, \mathrm{Sn}, \mathrm{S}, \mathrm{Se}, \mathrm{Mo}$, and $\mathrm{Cd}$. 
Table S1. Comparison of the characteristics of some reported flexible CZTSSe solar cells.

\begin{tabular}{llll}
\hline Material & Substrate & PCE $(\%)$ & Reference \\
\hline CZTSSe & Mo foil & 3.8 & {$[2]$} \\
CZTSe & Stainless steel foil & 6.1 & {$[3]$} \\
CZTS & Stainless steel foil & 6.29 & {$[4]$} \\
CZTSSe & Mo foil & 6.78 & {$[5]$} \\
CZTSSe & Mo foil & 8.0 & {$[6]$} \\
CZTSSe & Mo foil & 10.34 & {$[7]$} \\
\hline CZTS & Polyimide & 0.15 & {$[8]$} \\
CZTS & PET & 0.83 & {$[9]$} \\
CZTSSe & PET & 7.1 & this work \\
\hline
\end{tabular}

Table S2. Composition ratio of the CZTSSe absorber measured by EDX [10, 11].

\begin{tabular}{cccccc|ccc}
\hline & \multicolumn{4}{c|}{ Chemical composition } & \multicolumn{3}{c}{ Compositional ratio } \\
\cline { 2 - 9 } & $\begin{array}{c}\mathrm{Cu} \\
(\mathrm{at} \%)\end{array}$ & $\begin{array}{c}\mathrm{Zn} \\
(\mathrm{at} \%)\end{array}$ & $\begin{array}{c}\mathrm{Sn} \\
(\mathrm{at} \%)\end{array}$ & $\begin{array}{c}\mathrm{Se} \\
(\mathrm{at} \%)\end{array}$ & $\begin{array}{c}\mathrm{S} \\
(\mathrm{at} \%)\end{array}$ & $\begin{array}{c}\mathrm{Cu} / \\
(\mathrm{Zn}+\mathrm{Sn})\end{array}$ & $\mathrm{Zn} / \mathrm{Sn}$ & $\begin{array}{c}(\mathrm{Se}+\mathrm{S}) / \\
\mathrm{Metal}\end{array}$ \\
\hline $\begin{array}{l}\text { CZTSSe } \\
\text { absorber }\end{array}$ & 18 & 20.1 & 13.3 & 34.2 & 14.4 & 0.56 & 1.51 & 0.95 \\
\hline
\end{tabular}

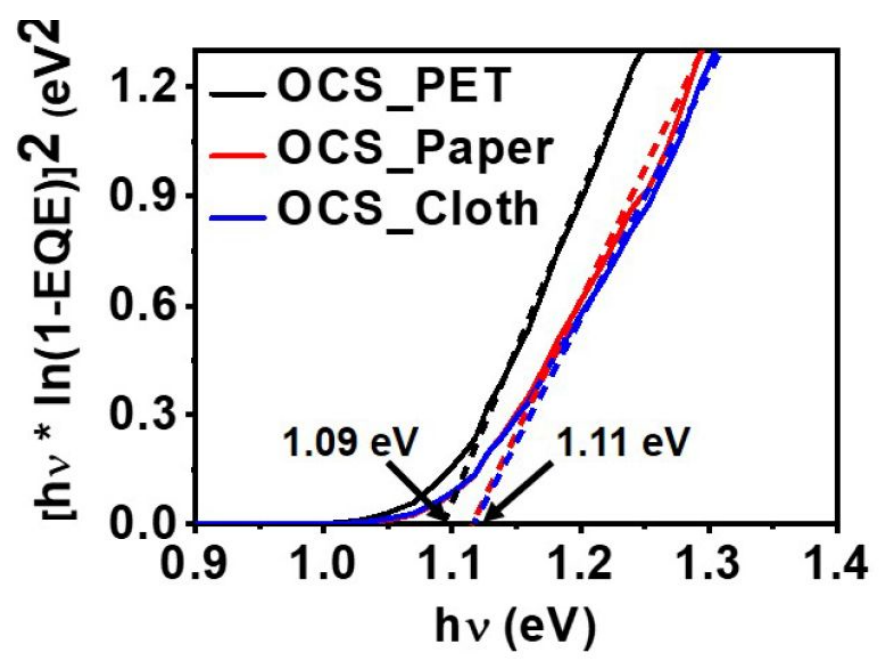

Figure S3. $[\mathrm{hv} \times \ln (1-\mathrm{EQE})]^{2}$ vs hv curves obtained from EQE measurement of the OCSs. 
Table S3. Device characteristics of the original CZTSSe solar cells on the soda-lime glasses for all cells.

\begin{tabular}{ccccccc}
\hline & $\begin{array}{c}\mathrm{V}_{\mathrm{oc}} \\
(\mathrm{mV})\end{array}$ & $\begin{array}{c}\mathrm{J}_{\mathrm{sc}} \\
\left(\mathrm{mA} / \mathrm{cm}^{2}\right)\end{array}$ & FF $(\%)$ & $\eta(\%)$ & $\begin{array}{c}\mathrm{R}_{\mathrm{sh}} \\
\left(\Omega \mathrm{cm}^{2}\right)\end{array}$ & $\begin{array}{c}\mathrm{R}_{\mathrm{s}} \\
\left(\Omega \mathrm{cm}^{2}\right)\end{array}$ \\
\hline OCS_PET 1 & 433.6 & 31.3 & 58.3 & 7.9 & 239.6 & 2.66 \\
OCS_PET 2 & 426 & 34.6 & 56.4 & 8.3 & 266.4 & 2.67 \\
OCS_PET 3 & 426 & 33.6 & 57.9 & 8.3 & 345 & 2.54 \\
OCS_PET 4 & 416 & 30.7 & 59.6 & 7.6 & 169.4 & 2.41 \\
OCS_PET 5 & 408.5 & 31.7 & 58.6 & 7.6 & 139.3 & 2.44 \\
OCS_PET 6 & 411.1 & 31.4 & 59.2 & 7.6 & 179 & 2.42 \\
\hline OCS_Paper 1 & 451.1 & 29.2 & 54.9 & 7.2 & 381 & 3.23 \\
OCS_Paper 2 & 441.1 & 31.8 & 53.8 & 7.6 & 315 & 3 \\
OCS_Paper 3 & 436.1 & 30.5 & 52.6 & 7.0 & 270.5 & 3.06 \\
OCS_Paper 4 & 443.6 & 29.5 & 51.3 & 6.7 & 282.6 & 3.4 \\
OCS_Paper 5 & 436.1 & 31.5 & 49.4 & 6.8 & 227.1 & 3.43 \\
OCS_Paper 6 & 223 & 27.9 & 31 & 1.9 & 11.7 & 5.1 \\
\hline OCS_Cloth 1 & 423.5 & 28.5 & 53.6 & 6.5 & 360 & 3.42 \\
OCS_Cloth 2 & 406 & 29.3 & 54 & 6.4 & 420 & 3.07 \\
OCS_Cloth 3 & 406 & 29.3 & 54.5 & 6.5 & 214.6 & 2.94 \\
OCS_Cloth 4 & 411.1 & 27.3 & 55.1 & 6.2 & 375 & 3.1 \\
OCS_Cloth 5 & 403.5 & 28.2 & 55.7 & 6.3 & 261 & 2.89 \\
OCS_Cloth 6 & 403.5 & 27.6 & 57.2 & 6.4 & 213.7 & 2.81 \\
\hline
\end{tabular}


(a)

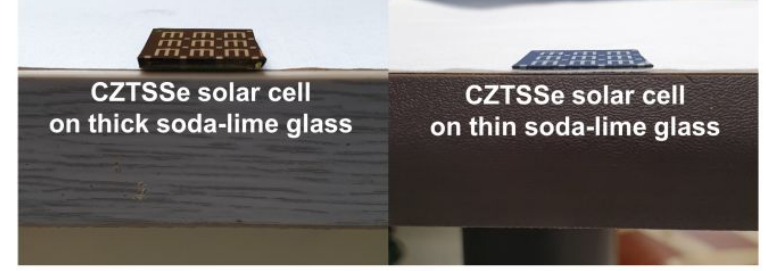

(b)

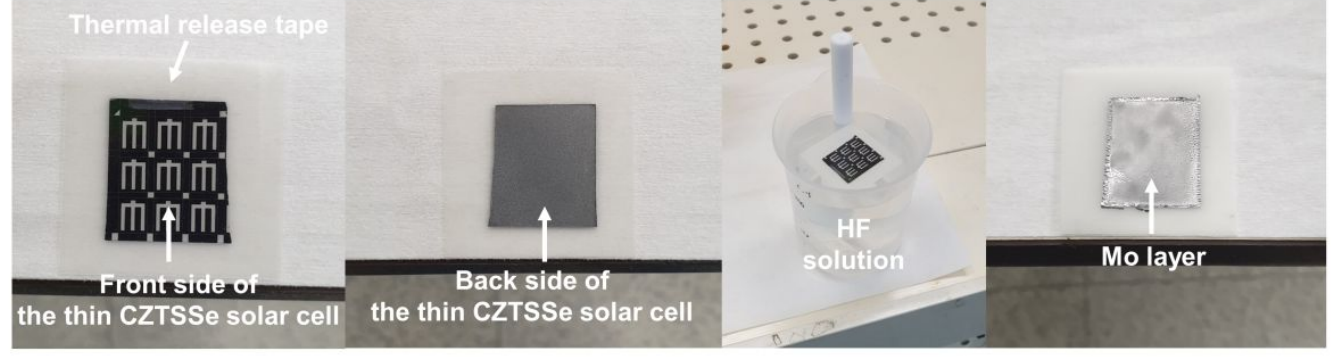

(c)

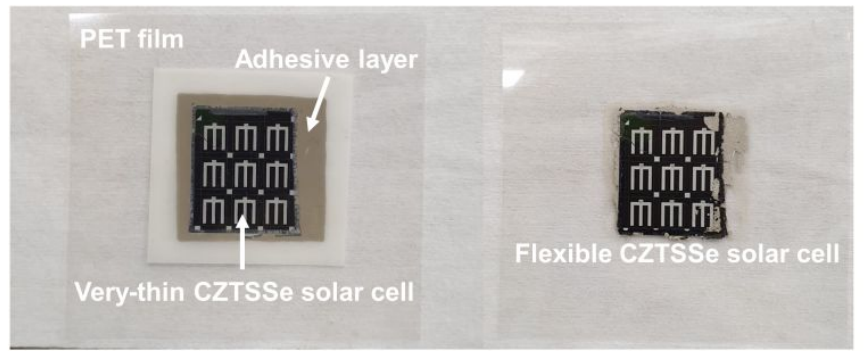

Figure S4. Digital camera images for fabrication process of a flexible CZTSSe solar cell on a PET substrate: (a) Grinding of soda-lime glass. (b) Removing whole sodalime glass. (c) Transferring the very-thin CZTSSe solar cell on the PET substrate. 


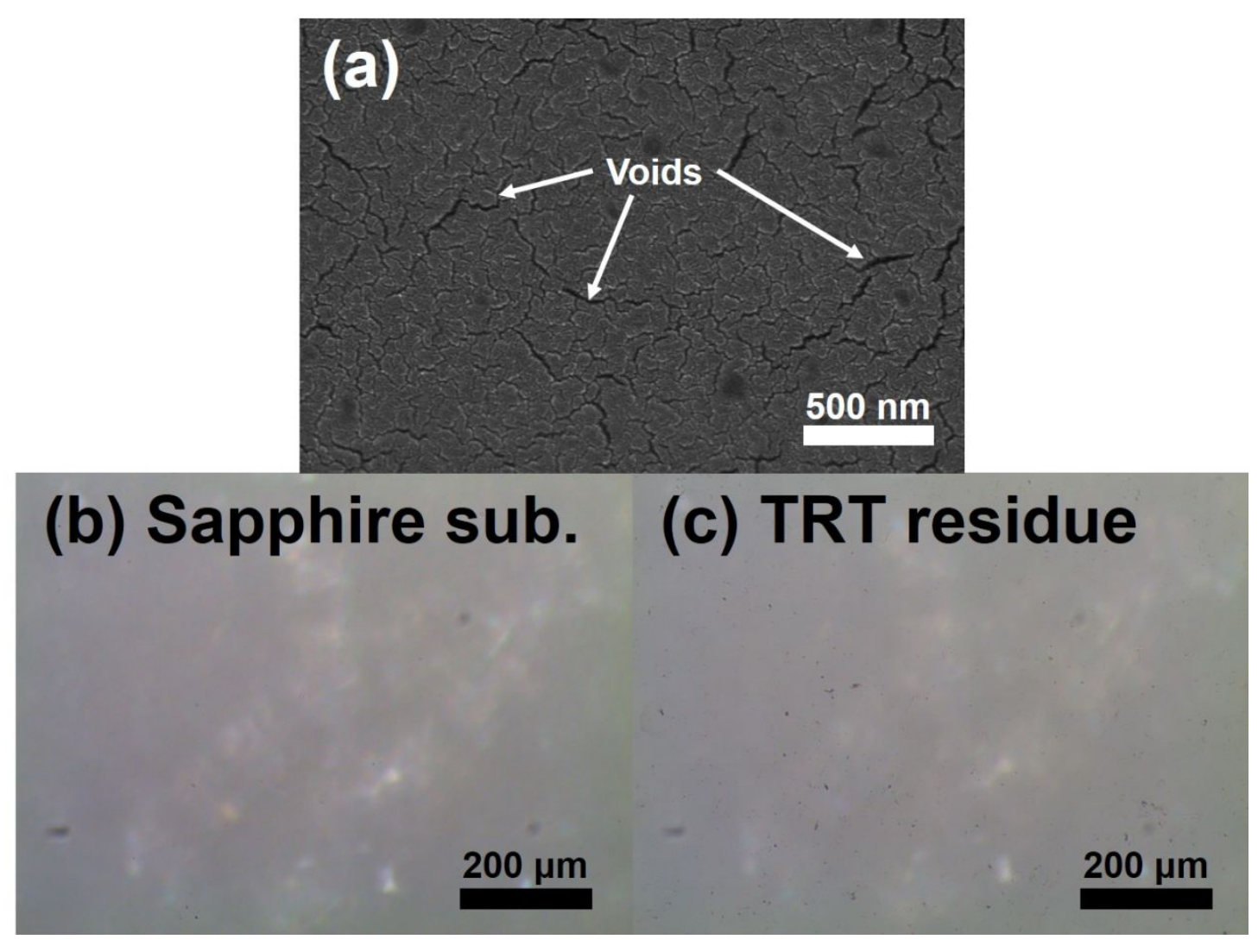

Figure S5. (a) An SEM image of the surface of the thermal release tape (TRT). Optical microscopy images of (b) the transparent sapphire substrate and (c) the TRT residue on it.

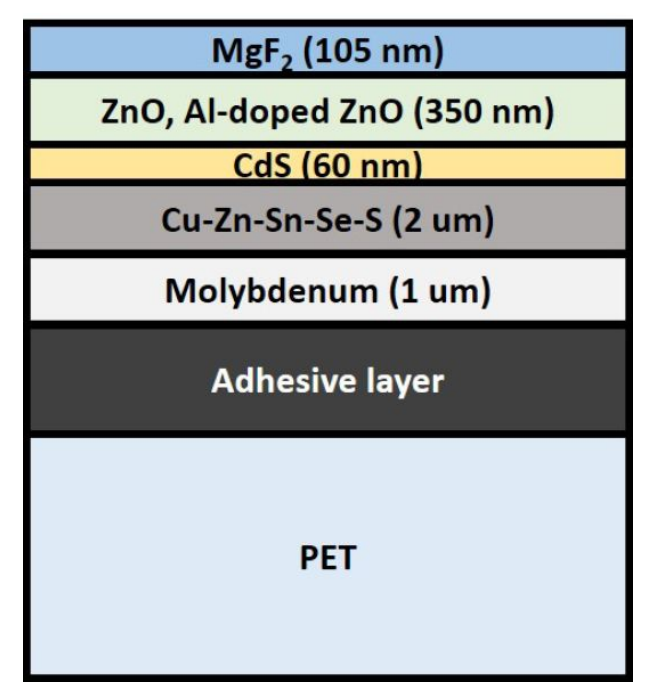

Figure S6. A schematic of the flexible CZTSSe solar cell fabricated on a PET substrate. 
Table S4. Device characteristics of the FCSs on various flexible substrates for all cells.

\begin{tabular}{lccccccc}
\hline & $\begin{array}{c}\mathrm{V}_{\mathrm{oc}} \\
(\mathrm{mV})\end{array}$ & $\begin{array}{c}\mathrm{J}_{\mathrm{sc}} \\
\left(\mathrm{mA} / \mathrm{cm}^{2}\right)\end{array}$ & $\begin{array}{c}\mathrm{FF} \\
(\%)\end{array}$ & $\begin{array}{c}\eta \\
(\%)\end{array}$ & $\begin{array}{c}\mathrm{R}_{\mathrm{sh}} \\
\left(\Omega \mathrm{cm}^{2}\right)\end{array}$ & $\begin{array}{c}\mathrm{R}_{\mathrm{s}} \\
\left(\Omega \mathrm{cm}^{2}\right)\end{array}$ & $\eta_{\mathrm{FCS}} / \eta_{\mathrm{OCS}}$ \\
\hline FCS_PET 1 & 428.6 & 25.2 & 49.3 & 5.3 & 167.5 & 4.26 & 0.67 \\
FCS_PET 2 & 423.6 & 28.2 & 49.8 & 5.9 & 176.4 & 3.35 & 0.71 \\
\hline FCS_PET 3 & 416.1 & 28.4 & 42.2 & 5 & 50.9 & 4.11 & 0.60 \\
FCS_PET 4 & 411.1 & 24 & 53 & 5.2 & 263.8 & 3.38 & 0.68 \\
FCS_PET 5 & 388.5 & 26.1 & 49.2 & 5 & 113.6 & 3.42 & 0.66 \\
FCS_PET 6 & 413.6 & 26.6 & 51.7 & 5.7 & 197.2 & 3.33 & 0.75 \\
\hline FCS_Paper 1 & 448.6 & 24.6 & 52.1 & 5.8 & 428.3 & 4.08 & 0.81 \\
FCS_Paper 2 & 436.1 & 27.7 & 51.7 & 6.2 & 251.6 & 3.49 & 0.82 \\
\hline FCS_Paper 3 & 418.6 & 25.7 & 50.9 & 5.5 & 247.2 & 3.57 & 0.79 \\
FCS_Paper 4 & 436.1 & 14.9 & 48.7 & 3.2 & 236.8 & 6.78 & 0.48 \\
FCS_Paper 5 & 418.6 & 26.6 & 46.8 & 5.2 & 152.1 & 4.23 & 0.76 \\
\hline FCS_Cloth 1 & 418.6 & 26.6 & 47.1 & 5.3 & 159 & 4.66 & 0.82 \\
FCS_Cloth 2 & 406 & 22.6 & 51.6 & 4.7 & 431.3 & 4.42 & 0.73 \\
FCS_Cloth 3 & 406 & 24.8 & 47.7 & 4.8 & 90.7 & 4.32 & 0.74 \\
FCS_Cloth 4 & 398.5 & 9.3 & 53.2 & 2 & 412 & 8.29 & 0.32 \\
FCS_Cloth 5 & 391 & 12 & 54.9 & 2.6 & 519.2 & 6.3 & 0.41 \\
FCS_Cloth 6 & 401 & 26.7 & 53.9 & 5.8 & 150.3 & 3.63 & 0.91 \\
\hline
\end{tabular}




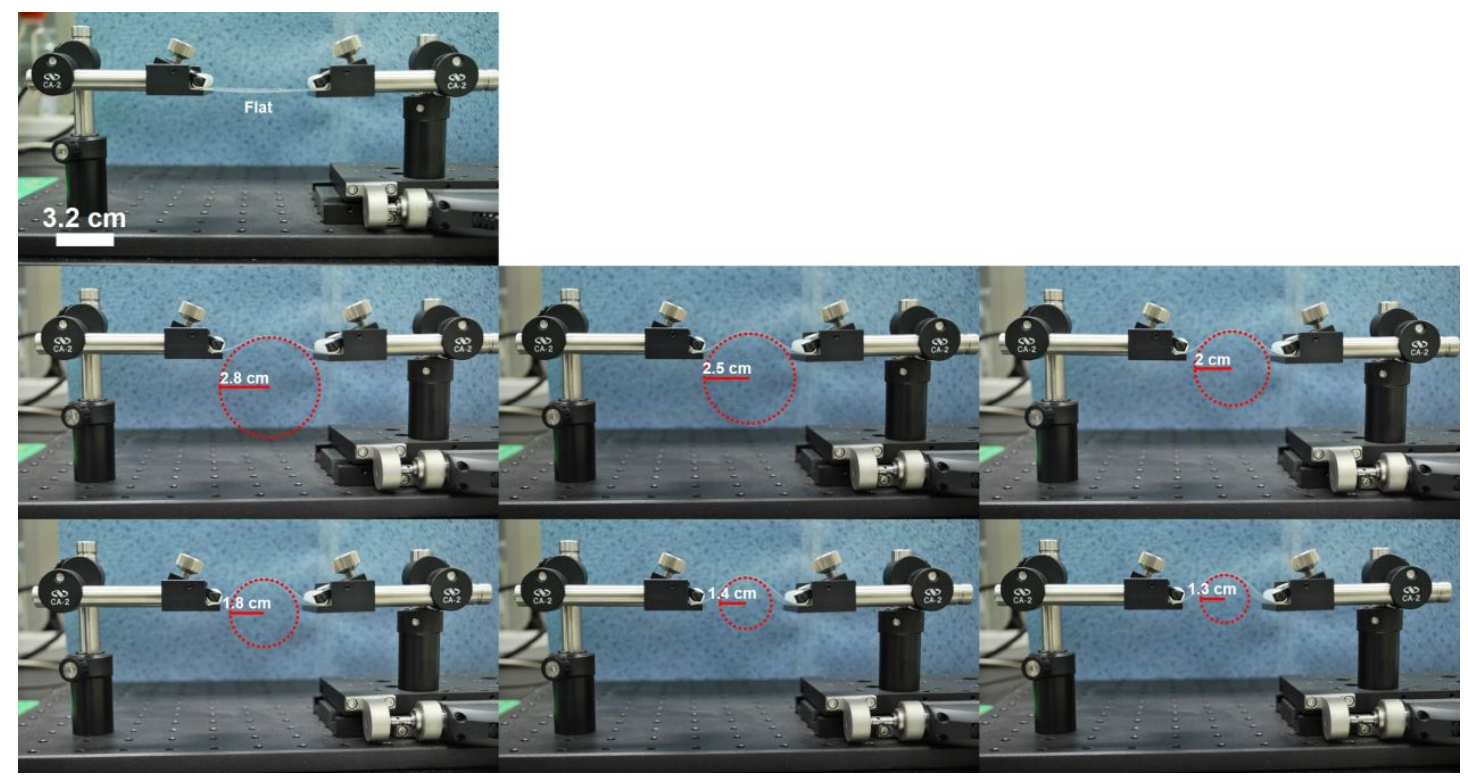

Figure S7. Bending tests by changing the bending radius from $2.8 \mathrm{~cm}$ to $1.3 \mathrm{~cm}$.

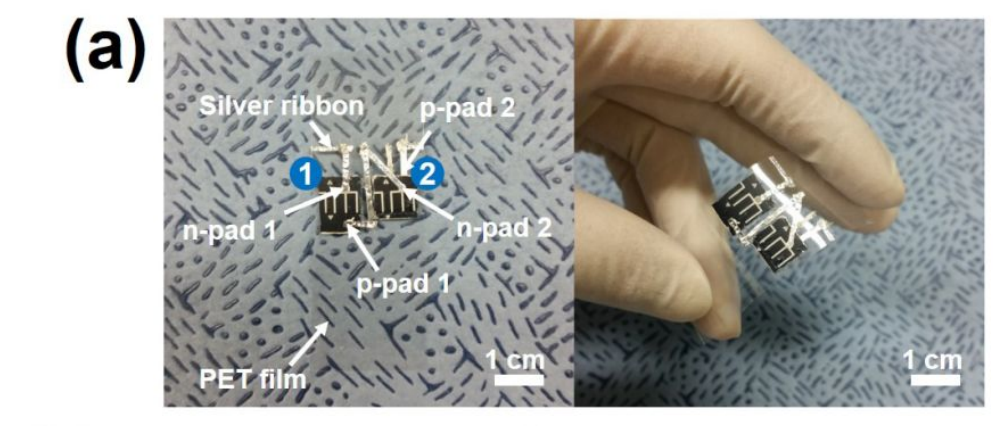

(b)
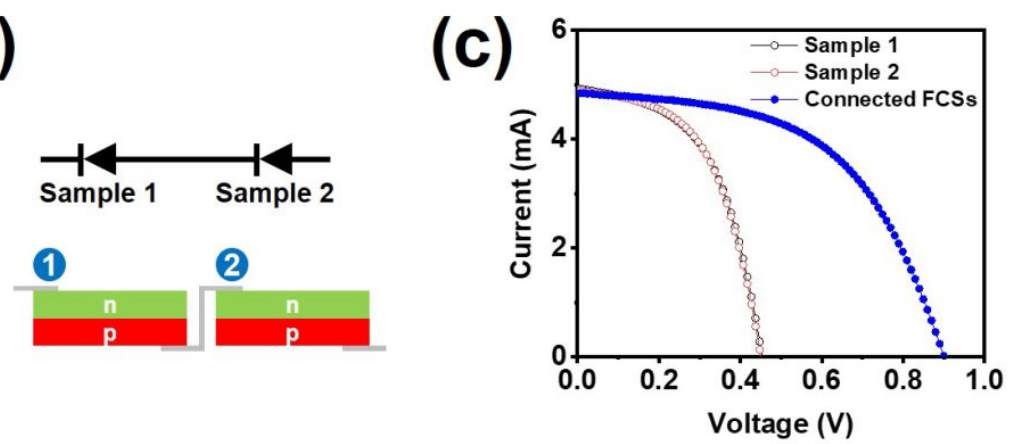

Figure S8. Conveniently connected FCSs: (a) Digital camera images of the conveniently connected FCSs. (b) Schematics of the series-connected solar cells. (c) J$\mathrm{V}$ characteristics for two FCSs and the connected FCSs. 
Table S5. Solar cell characteristics of the two FCSs and conveniently connected FCSs.

\begin{tabular}{cccccccc}
\hline & $\begin{array}{c}\mathrm{V}_{\mathrm{oc}} \\
(\mathrm{mV})\end{array}$ & $\begin{array}{c}\mathrm{I}_{\mathrm{sc}} \\
(\mathrm{mA})\end{array}$ & $\begin{array}{c}\mathrm{J}_{\mathrm{sc}} \\
\left(\mathrm{mA} / \mathrm{cm}^{2}\right)\end{array}$ & $\begin{array}{c}\mathrm{FF} \\
(\%)\end{array}$ & $\begin{array}{c}\eta \\
(\%)\end{array}$ & $\begin{array}{c}\mathrm{R}_{\mathrm{sh}} \\
\left(\Omega \mathrm{cm}^{2}\right)\end{array}$ & $\begin{array}{c}\mathrm{R}_{\mathrm{s}} \\
\left(\Omega \mathrm{cm}^{2}\right)\end{array}$ \\
\hline Sample 1 & 457.3 & 4.9 & 25.9 & 52.2 & 6.18 & 141.7 & 4.2 \\
Sample 2 & 447.2 & 4.9 & 25.7 & 53.9 & 6.19 & 172.3 & 4.47 \\
$\begin{array}{c}\text { Connected } \\
\text { FCSs }\end{array}$ & 899.5 & 4.8 & 12.7 & 53.6 & 6.1 & 787.4 & 17.13 \\
\hline
\end{tabular}

Table S6. Device characteristics of the extra CZTSSe solar cell sample for all cells.

\begin{tabular}{cccccccc}
\hline & $\begin{array}{c}\mathrm{V}_{\mathrm{oc}} \\
(\mathrm{mV})\end{array}$ & $\begin{array}{c}\mathrm{J}_{\mathrm{sc}} \\
\left(\mathrm{mA} / \mathrm{cm}^{2}\right)\end{array}$ & $\begin{array}{c}\mathrm{FF} \\
(\%)\end{array}$ & $\begin{array}{c}\eta \\
(\%)\end{array}$ & $\begin{array}{c}\mathrm{R}_{\mathrm{sh}} \\
\left(\Omega \mathrm{cm}^{2}\right)\end{array}$ & $\begin{array}{c}\mathrm{R}_{\mathrm{s}} \\
\left(\Omega \mathrm{cm}^{2}\right)\end{array}$ & $\begin{array}{c}\eta_{\mathrm{FCS}}{ }^{\prime} \\
\eta_{\mathrm{OCS}}\end{array}$ \\
\hline $\begin{array}{c}\text { OCS_PET } \\
\text { extra 1 }\end{array}$ & 458.7 & 30.9 & 54.8 & 7.8 & 168.6 & 3.42 & - \\
$\begin{array}{c}\text { OCS_PET } \\
\text { extra 2 }\end{array}$ & 458.7 & 32.2 & 58.4 & 8.6 & 224.4 & 3.05 & - \\
$\begin{array}{c}\text { OCS_PET } \\
\text { extra 3 }\end{array}$ & 458.7 & 31.3 & 55.9 & 8.0 & 190.1 & 3.31 & - \\
$\begin{array}{c}\text { OCS_PET } \\
\text { extra 4 }\end{array}$ & 448.6 & 32 & 56.3 & 8.1 & 211.1 & 3.21 & - \\
$\begin{array}{c}\text { OCS_PET } \\
\text { extra 5 }\end{array}$ & 463.7 & 31.4 & 59.7 & 8.7 & 235.8 & 2.89 & - \\
$\begin{array}{c}\text { OCS_PET } \\
\text { extra 6 }\end{array}$ & 468.7 & 34.4 & 57.6 & 9.3 & 175.7 & 2.81 & - \\
\hline $\begin{array}{c}\text { FCS_PET } \\
\text { extra 1 }\end{array}$ & 453.6 & 28.9 & 54.3 & 7.1 & 106.5 & 3.29 & 0.91 \\
$\begin{array}{c}\text { FCS_PET } \\
\text { extra 2 }\end{array}$ & 448.6 & 26.6 & 49.7 & 5.9 & 102.7 & 4.85 & 0.69 \\
$\begin{array}{c}\text { FCS_PET } \\
\text { extra 3 }\end{array}$ & 448.6 & 27.5 & 54 & 6.7 & 137.4 & 3.89 & 0.84 \\
$\begin{array}{c}\text { FCS_PET } \\
\text { extra 4 }\end{array}$ & 443.6 & 27.1 & 52.4 & 6.3 & 134.8 & 4.45 & 0.78 \\
$\begin{array}{l}\text { FCS_PET } \\
\text { extra 5 }\end{array}$ & 453.6 & 24.9 & 57.3 & 6.5 & 188.4 & 3.59 & 0.75 \\
$\begin{array}{c}\text { FCS_PET } \\
\text { extra 6 }\end{array}$ & 453.6 & 23.8 & 41.4 & 4.5 & 111.7 & 9.07 & 0.48 \\
\hline
\end{tabular}


(a)

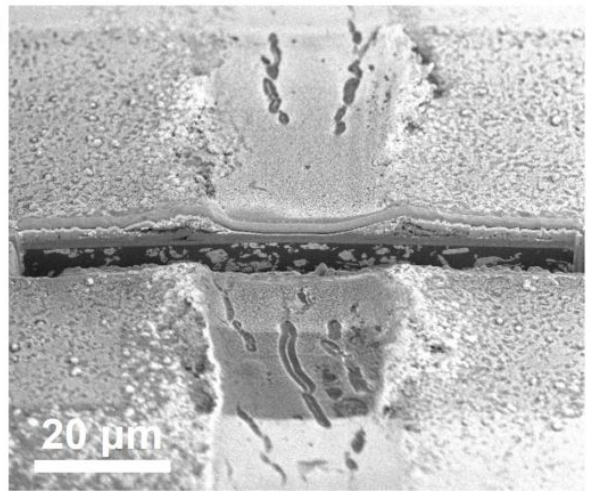

(b)

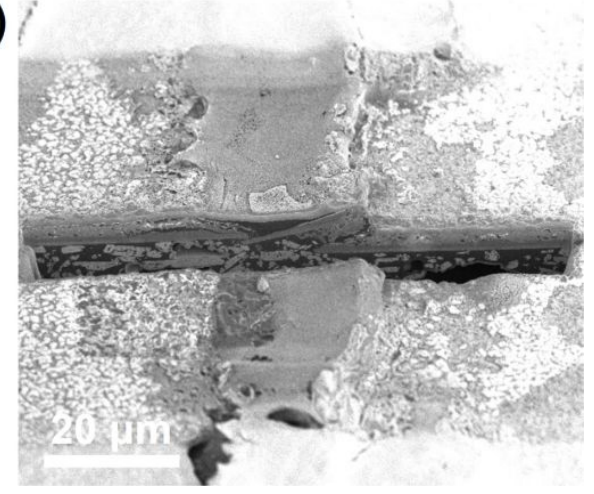

Figure S9. (a) and (b) indicate the zoom-out FIB images for the two boundaries.

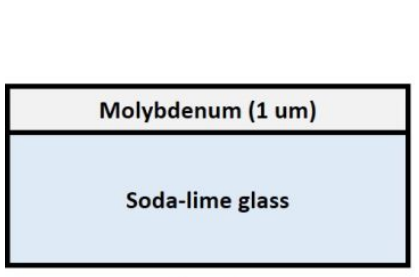

(a)

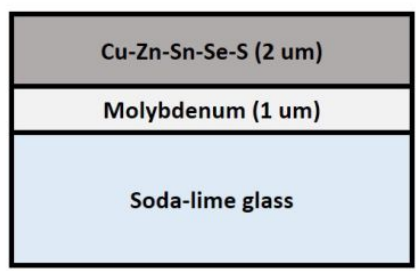

(b)

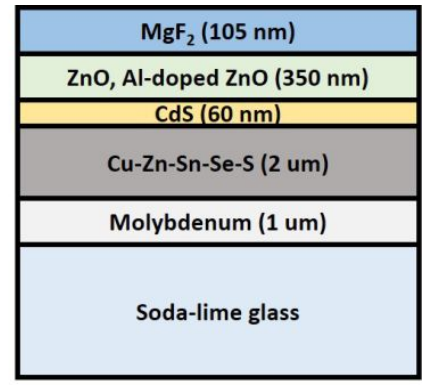

(c)

Figure S10. Schematic of each measured layer for nanoindentation [12]. (a) Mo layer on soda-lime glass (Mo layer). (b) CZTSSe absorber on Mo/soda-lime glass (Absorber). (c) CZTSSe solar cell including $\mathrm{MgF}_{2}$ layer (CZTSSe solar cell).

Table S7. Results of hardness and Young's modulus measured by nanoindentation.

\begin{tabular}{ccc}
\hline Materials & Hardness (Gpa) & Young's modulus (Gpa) \\
\hline Razor blade & 9.9 & 223.5 \\
Mo & 9.1 & 162 \\
Absorber & 2.3 & 53 \\
CZTSSe solar cell & 4.1 & 72.8 \\
\hline
\end{tabular}




\section{References}

(1) Chen, W.C.; Tunuguntla, V.; Li, H.W.; Chen, C.Y.; Li, S.S.; Hwang, J.S.; Lee, C.H.; Chen, L.C.; Chen, K.H. Fabrication of $\mathrm{Cu}_{2} \mathrm{ZnSnSe}_{4}$ solar cells through multi-step selenization of layered metallic precursor film. Thin Solid Films 2016, 618, 42-49.

(2) Xu, X.; Qu, Y.; Barrioz, V.; Zoppi, G.; Beattie, N.S. Reducing series resistance in $\mathrm{Cu}_{2} \mathrm{ZnSn}(\mathrm{S}, \mathrm{Se})_{4}$ nanoparticle ink solar cells on flexible molybdenum foil substrates. RSC Adv. 2018, 8, 3470-3476.

(3) López-Marino, S.; Sánchez, Y.; Espíndola-Rodríguez, M.; Alcobé, X.; Xie, H.; Neuschitzer, M.; Becerril, I.; Giraldo, S.; Dimitrievska, M.; Placidi, M.; Fourdrinier, L.; Izquierdo-Roca, V.; Pérez-Rodríguez, A.; Saucedo, E. Alkali doping strategies for flexible and light-weight $\mathrm{Cu}_{2} \mathrm{ZnSnSe}_{4}$ solar cells. J. Mater. Chem. A 2016, 4, $1895-$ 1907.

(4) Sun, K.; Liu, F.; Huang, J.; Yan, C.; Song, N.; Sun, H.; Xue, C.; Zhang, Y.; Pu, A.; Shen, Y.; Stride, J.A.; Green, M.; Hao, X. Flexible kesterite $\mathrm{Cu}_{2} \mathrm{ZnSnS}_{4}$ solar cells with sodium-doped molybdenum back contacts on stainless steel substrates. Sol. Energy Mater. Sol. Cells 2018, 182, 14-20.

(5) Yan, Q.; Cheng, S.; Li, H.; Yu, X.; Fu, J.; Tian, Q.; Jia, H.; Wu, S. High flexible $\mathrm{Cu}_{2} \mathrm{ZnSn}(\mathrm{S}, \mathrm{Se})_{4}$ solar cells by green solution-process. Sol. Energy 2019, 177, 508-516.

(6) Jo, E.; Gang, M.G.; Shim, H.; Suryawanshi, M.P.; Ghorpade, U.V.; Kim, J.H. 8\% efficiency $\mathrm{Cu}_{2} \mathrm{ZnSn}(\mathrm{S}, \mathrm{Se})_{4}(\mathrm{CZTSSe})$ thin film solar cells on flexible and lightweight molybdenum foil substrates. ACS Appl. Mater. Interfaces 2019, 11, 23118-23124.

(7) Yang, K.; Kim, S.; Kim, S.; Ahn, K.; Son, D.; Kim, S.; Lee, S.; Kim, Y.; Park, S.; Sung, S.; Kim, D.; Enkhbat, T.; Kim, J.; Jeon, C.; Kang, J. Flexible $\mathrm{Cu}_{2} \mathrm{ZnSn}(\mathrm{S}, \mathrm{Se})_{4}$ solar cells with over $10 \%$ efficiency and methods of enlarging the cell area. Nat. Commun. 2019, 10, 1-10.

(8) Boshta, M.; Binetti, S.; Donne, A.L.; Gomaa, M; Acciarri, M. A chemical deposition process for low-cost- CZTS solar cell on flexible substrates. Mater. Tech. 2016, 32, 251-255.

(9) Najafi, V.; Kimiagar, S. Cd-free $\mathrm{Cu} 2 \mathrm{ZnSnS} 4$ thin film solar cell on a flexible substrate using nano-crystal ink. Thin solid Films 2018, 657, 70-75. 
(10) Wang, W.; Winkler, M.T.; Gunawan, O.; Gokmen, T.; Todorov, T.K.; Zhu, Y.; Mitzi, D.B. Device characteristics of CZTSSe thin-film solar cells with $12.6 \%$ efficiency. Adv. Energy Mater. 2014, 4, 1301465.

(11) Yang, K.; Son, D.; Sung, S.; Sim, J.; Kim, Y.; Park, S.; Jeon, D.; Kim, J.; Hwang, D.; Jeon, C.; Nam, D.; Cheong, H.; Kang, J.; Kim, D. A band-gap-graded CZTSSe solar cell with $12.3 \%$ efficiency. J. Mater. Chem. A 2016, 4, 10151-10158.

(12) Malerba, C.; Valentini, M.; Azanza Rcardo, C.L.; Rinaldi, A.; Cappelletto, E.; Scardi, P.; Mittiga, A. Blistering in $\mathrm{Cu}_{2} \mathrm{ZnSnS}_{4}$ thin films: correlation with residual stresses. Mater. Des. 2016, 108, 725-735. 